Supplement Issue 1: International Tehran Breast Cancer Congress (TBCC9)

\title{
Development of anti-FZD7 scFv Antibody and Evaluation of its Apoptotic Effect on Breast Cancer Cells
}

DOI: $10.21859 /$ mci-supp-37

\author{
Neda Zarei ${ }^{1,}$, , Foroogh Nejatollahi ${ }^{2,3}$ \\ ${ }^{1}$ Biotechnology section, Department of Pathobiology, School of Veterinary Medicine, \\ Shiraz University, Shiraz, Iran \\ ${ }^{2}$ Shiraz HIV/AIDS Research Center, Institute of Health, Shiraz University of \\ Medical Sciences, Shiraz, Iran \\ ${ }^{3}$ Recombinant Antibody Laboratory, Department of Immunology, Shiraz University \\ of Medical Sciences, Shiraz, Iran \\ * Corresponding author: Neda Zarei, Biotechnology section, Department of \\ Pathobiology, School of Veterinary Medicine, Shiraz University, Shiraz, Iran. E-mail: \\ zareined@gmail.com
}

\section{Keywords:}

Anti-FZD scFv

Frizzled Receptor 7

Breast Cancer

\begin{abstract}
Introduction Frizzled family receptor 7 (FZD7) is one of the 10 members of Frizzled receptors. It interacts with Wnt ligands to activate canonical wnt signaling, which turns on different downstream transcription factors essential for modulating cellular proliferation, polarity, and differentiation. Altered expression of FZD7 receptor is associated with development and progression of many cancers including breast cancer. An effective targeted therapy for breast cancer through modulating ligand-receptor interaction may involve the use of antibodies to antagonize FZD7. ScFvs (single-chain fragment variable) have provided an alternative to full-length monoclonal antibodies (mAbs) in diagnostic and therapeutic applications.

Materials and Methods: A phage antibody library of scFv was used and selection of specific scFvs were performed by 4 rounds of panning process against an immunodominant epitope of FZD7, followed by PCR and fingerprinting of the selected clones. ELISA was used to confirm the specificity of the clones. Apoptotic effects of the selected scFv on MDAMB-231 cell line were assessed by annexin V/PI assay after $24 \mathrm{~h}$ and $48 \mathrm{~h}$.

Results: A specific scFv with the frequency of $35 \%$ was isolated which produced positive ELISA with the corresponding epitope. After $24 \mathrm{~h}$ treatment with the selected scFv, MDA-MB-231 cells showed 48.7\% apoptotic cell death (Annexin V+/PI-). However, this amount increased to $81.6 \%$ following $48 \mathrm{~h}$ treatment with scFv.

Conclusions: Due to unique apoptotic properties of selected $s c F v$ including human origin, high affinity and specificity, this agent has been applied in cancer immunotherapy. The specific anti-FZD7 scFv selected in this study has the potential to be used for inhibiting wnt signaling pathway in breast cancer cells.
\end{abstract}

